\title{
Positive Effects of Ionic Liquids in the Oxidative Cleavage of vic-diols Catalyzed by Mn(III) Complexes
}

\author{
D. Fernández, S. Riaño and L. Fadini ${ }^{*}$
}

Departamento de Química, Facultad de Ciencias, Universidad Nacional de Colombia, Cra 30 No. 45-03, Bogotá D.C. Colombia

\begin{abstract}
The obtaining of aldehydes or ketones through oxidative cleavage of vicinal diols requires the use of a catalyst. In this work we present the positive effects of ionic liquids in the homogeneous oxidative cleavage of ethylenglycol, pinacol, benzopinacol and hydrobenzoin, catalyzed by $\mathrm{Mn}(\mathrm{salen})$ complexes. The catalyst dissolved in ionic liquids allows high efficiency (yield up to $99 \%$ with air as oxidant), low environmental impact and, in addition, it is possible to recycle.

In order to understand the role of the catalyst and of the ionic liquids, DFT studies were performed and a possible catalytic cycle is presented.
\end{abstract}

\section{INTRODUCTION}

The oxidative cleavage of vicinal diols to obtain aldehydes is an important field in synthetic organic chemistry [1], and also for the pharmaceutical industry [2]. Nonetheless, the reported stoichiometric [3-5] and catalytic [6-8] systems are far away from the complete accomplishment in terms of activity, selectivity, atomic economy and environmental impact. Therefore, the development of new catalytic systems that allow the selective formation of aldehydes with non-polluting and non-toxic catalysts and/or oxidants, is one of the aims of the organic synthesis [9]. Our approach is based on the use of ionic liquids (organic salts with low melting-point $(<100$ $\left.\left.{ }^{\circ} \mathrm{C}\right)\right)[10,11]$ and the well-known achiral $\mathrm{Mn}^{\mathrm{III}}$ (salen) complexes ((N,N'-bis(salicylidene)-1,2-ethylendiamine) manganese(III)) [12-14], - which have been intensely investigated owing to their excellent performance in alkene epoxidation - as a catalytic system for the oxidative C-C bond cleavage of vic-diols to aldehydes with oxygen as oxidant and a sacrificial aldehyde as a co-reductant (Scheme 1) [15]. The highly polar ionic liquids (IL) can stabilize the cationic catalyst or the transition states [16], having as a consequence an increased activity of the catalyst [17, 18]. Additionally, the possibility to recycle the catalyst/IL system as a strategy to improve one of the deficiency of the homogeneous catalysts allows also an improved total TON (turnover number) [19].

\section{Experimental Part}

The routine ${ }^{13} \mathrm{C}\left\{{ }^{1} \mathrm{H}\right\}$-, and ${ }^{1} \mathrm{H}-\mathrm{NMR}$ spectra were measured in the given solvent on either a Bruker Avance 400 at room temperature. The chemical shifts $\delta$ are given in ppm relative to TMS, and referenced to the solvent signal for ${ }^{1} \mathrm{H}-$

*Address correspondence to this author at the Departamento de Química, Facultad de Ciencias, Universidad Nacional de Colombia, Cra 30 No. 45-03, Bogotá D.C. Colombia; Fax: +57 13165220 ; E-mail: 1fadini@unal.edu.co and ${ }^{13} \mathrm{C}\left\{{ }^{1} \mathrm{H}\right\}-N M R$. The FT-IR were measured on a Perkin Elmer Paragon 500. GC-MS-analysis were done with a
ThemoFinnigan TraceMS, with EI-MS as detector; column: Zebron ZB-5 (30m x $0.25 \mathrm{~mm} \times 0.25 \mu \mathrm{m})$. All reagents (Merck or Aldrich) were used as received (LabGrade). The catalytic substrates and the catalyst were synthesized following reported procedure in literature: (+/-)1,2diphenyl-1,2-ethanediol [20], 1,1,2,2-tetraphenyl-1,2-ethanediol [21], 2,3-dimethyl- 2,3-butanediol [22]. The ionic liquids (Solvent Innovation $\mathrm{GmbH}$, LabGrade, 99\% purity) were used as received. The recycled attempts were done in a parallel organic synthesizer Quest 210 (Artisian Scientific), with $20^{*} 5 \mathrm{~mL}$ vials and vertical stirring, under air (1 bar). The extraction of the products were done with $10 * 3 \mathrm{~mL}$ of hexane:ethyl acetate $(9: 1)$. The isolated products were analyzed by ${ }^{1} \mathrm{H}-\mathrm{NMR}$, GC-MS and compared with literature.

Standard catalysis procedure: $0.27 \mathrm{mmol}$ of the diol is dissolved in $2 \mathrm{~mL}$ of dichloromethane and added to a round-bottomed flask that contains $5.0 \mathrm{~mL}$ of the ionic liquid and $0.014 \mathrm{mmol}(5 \mathrm{~mol} \%)$ of $[\mathrm{Mn}(\mathrm{salen})(\mathrm{Py})](\mathrm{OAc})$. The organic solvent is evaporated and the system is bubbled constantly with oxygen at $30{ }^{\circ} \mathrm{C}$. One equivalent of the sacrificial aldehyde $(0.27 \mathrm{mmol}$, benzaldehyde or salicylaldehyde) is added and the addition is repeated after 1 and 2 hours. After three hours, the air bubbling is suspended and $5 \mathrm{~mL}$ of hexane are added to the system. The products are extracted $(10 * 5 \mathrm{~mL})$ and purified (flash chromatography) with hexane/ethylacetate $9: 1$ or distilled under reduced pressure. The yields are for isolated products.

Standard catalysis procedure in Quest 210 (20 parallel vials): a standard solution of $2.7 \mathrm{mmol}$ of the diol is dissolved in $20 \mathrm{~mL}$ of dichloromethane and $2 \mathrm{~mL}$ of this standard solution is added to $5 \mathrm{~mL}$ vial that contains $2.0 \mathrm{~mL}$ of the ionic liquid and $0.014 \mathrm{mmol}$ (5 mol\%) of $[\mathrm{Mn}($ salen $)(\mathrm{Py})](\mathrm{OAc})$. After the evaporation of the organic solvent, $0.81 \mathrm{mmol}$ of the sacrificial benzaldehyde are added and the system is bubbled constantly with air at $60{ }^{\circ} \mathrm{C}$. After three hours, the air bubbling is suspended and $3 \mathrm{~mL}$ of hexane are added to the system. The products are extracted $\left(10^{*} 3 \mathrm{~mL}\right.$ ) and purified (flash chromatography) with hexane/ethylacetate $9: 1$. The yields are for isolated products. 
catalyst:

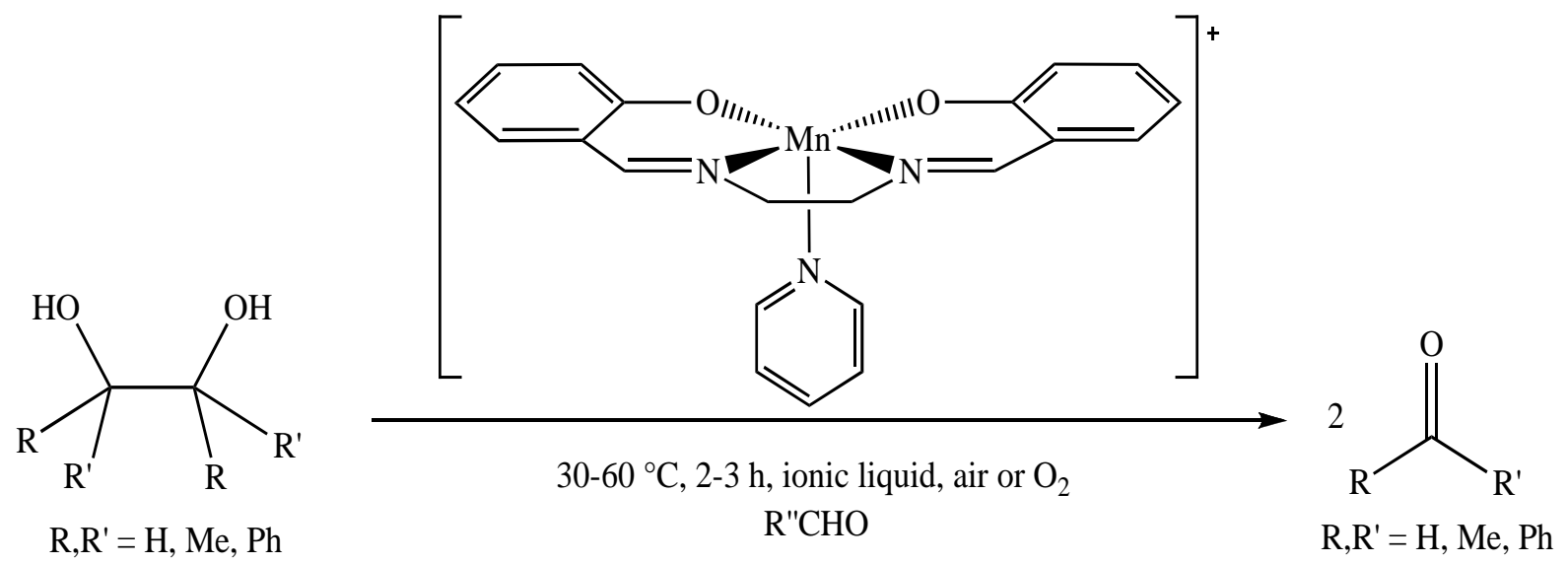

Scheme 1. Oxidative cleavage of vic-diols catalyzed by Mn(III)-complexes.

\section{RESULTS AND DISCUSSION}

The tested diols in the previous reported Mn-catalyzed oxidative-cleavage in ionic liquids [23] were 1,2-ethanediol (ethylenglycol, R=R'=H), 2,3-dimethyl-2,3-butanediol (pinacol, $\mathrm{R}=\mathrm{R}^{\prime}=\mathrm{Me}$ ), 1,2-diphenyl-1,2-ethanediol (hydrobenzoin, $\mathrm{R}=\mathrm{Ph}, \mathrm{R}^{\prime}=\mathrm{H}$ ) and 1,1,2,2-tetraphenyl-1,2-ethanediol (benzopinacol, $\mathrm{R}=\mathrm{R}{ }^{\prime}=\mathrm{Ph}$ ) (Scheme 1), so we could establish differences of reactivity as regards electronic and steric effects. The used ionic liquids were derivatives of imidazolium and picolinium salts (Scheme 2). Fig. (1) shows the results of the preliminary catalytic experiments (reaction conditions: $3 \mathrm{~h}, 30{ }^{\circ} \mathrm{C}$, with constant bubbling of oxygen, 5 mol\% of catalyst (in situ or isolated) and benzaldehyde or salicylaldehyde as sacrificial aldehyde) [23].

The catalytic activity of the Mn-complexes toward the oxidative cleavage of vicinal diols showed better results in ionic liquids than in organic solvents. The best oxidation condition was the use of oxygen as an oxidant in the presence of excess aldehyde as a sacrificial reagent, the so-called Mukaiyama catalytic system [15, 24].

Also in the in situ system (the catalyst is formed from 5\% mol of $\mathrm{Mn}(\mathrm{OAc})_{2}$ with 1 equivalent of $\mathrm{N}, \mathrm{N}$ '-bis(salicylidene)-1,2-ethylendiamine (= salen), and 1 equivalent of pyridine dissolved in dichloromethane/ionic liquid) there is improved activity if the reaction is carried out in ionic liquids as a solvent (entry 1 of Fig. (1), increasing of the yield from $18.4 \%$ in dichloromethane to $60.1 \%$ in $\left.[\mathrm{OPIC}]\left[\mathrm{PF}_{6}\right]\right)$. The use of the isolated catalyst $[\mathrm{Mn}($ salen $)(\mathrm{Py})](\mathrm{OAc})$ allowed us to obtain better yields (77.8\% in dichloromethane and $>99 \%$ in $[\mathrm{OPIC}]\left[\mathrm{PF}_{6}\right]$, entry 2 ). This result is definitively better than that reported by
Pedro and coworkers, using the o-phenylendioxamate $\mathrm{Mn}^{\mathrm{III}}$ catalyst (in acetonitrile, at $40{ }^{\circ} \mathrm{C}$, after $6 \mathrm{~h}$ : $10 \%$ yield) [25]. Although the role of the sacrificial aldehyde has not been clearly established, the Mukaiyama group proposed that for aerobic epoxidation reactions in the presence of this type of complex the reaction proceeds simultaneously with the co-oxidation of the aldehyde, forming an oxo intermediate $[15,24]$. We therefore evaluated the effect of two sacrificial aldehydes in the oxidative cleavage reaction of benzopinacol: benzaldehyde and salicylaldehyde. The yields using benzaldehyde as co-reductant always showed better results (entries $2 v s 3$ ), indicating the important role of this aldehyde in the formation of the active species. The oxidative cleavage of 2,3-dimethyl-2,3-butanediol allowed the formation of acetone in moderate to good yields when the catalysis was carried out in dichloromethane $(43.2 \%)$, in $[\mathrm{BMIM}]\left[\mathrm{PF}_{6}\right](58.5 \%)$ or in $\left.[\mathrm{OPIC}] \mathrm{PF}_{6}\right](77,9 \%$; entry 4$)$. On the other hand, the results with ethylenglycol to form formaldehyde showed only low yields (up to $30.2 \%$ ), and no appreciative amounts of the corresponding acid have been detected (entry 5). In the oxidative cleavage of 1,2-diphenyl-1,2-ethanediol, the formation of benzaldehyde was slightly superior: $17.1 \%$ in dichloromethane and close to $40 \%$ in the ionic liquids (entry 6), comparable with the yields reported by Pedro et al. [25], with only traces of the corresponding acid.

In order to extend this reaction catalyzed by $\mathrm{Mn}$ (III) complexes in a range of ionic liquids, the oxidative cleavage of benzopinacol have been tried in different imidazoliumand picolinium-based ionic liquids (Fig. 2).

The aerobic oxidative cleavage of benzopinacol at $60{ }^{\circ} \mathrm{C}$ with benzaldehyde as co-reductant and air (instead of pure

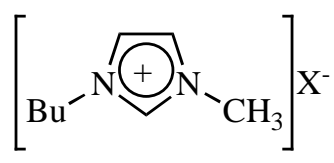

[BMIM]X

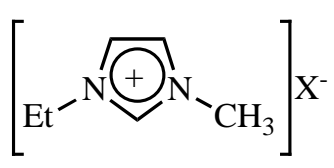

[EMIM]X<smiles></smiles>

[OPIC]X

Scheme 2. Used ionic liquids: [BMIM]X, [EMIM]X and [OPIC]X, where $\mathrm{X}$ is the anion $\mathrm{PF}_{6}, \mathrm{BF}_{4}, \mathrm{CF}_{3} \mathrm{SO}_{3}$ or $\mathrm{N}(\mathrm{CN})_{2}(\mathrm{DCA})$ and $[\mathrm{BMIM}]=$ $N$-butyl- $N$-methylimidazolium salts; [EMIM] $=N$-ethyl- $N$-methylimidazolium salts; [OPIC] $=N$-octyl-picolinium salts. 


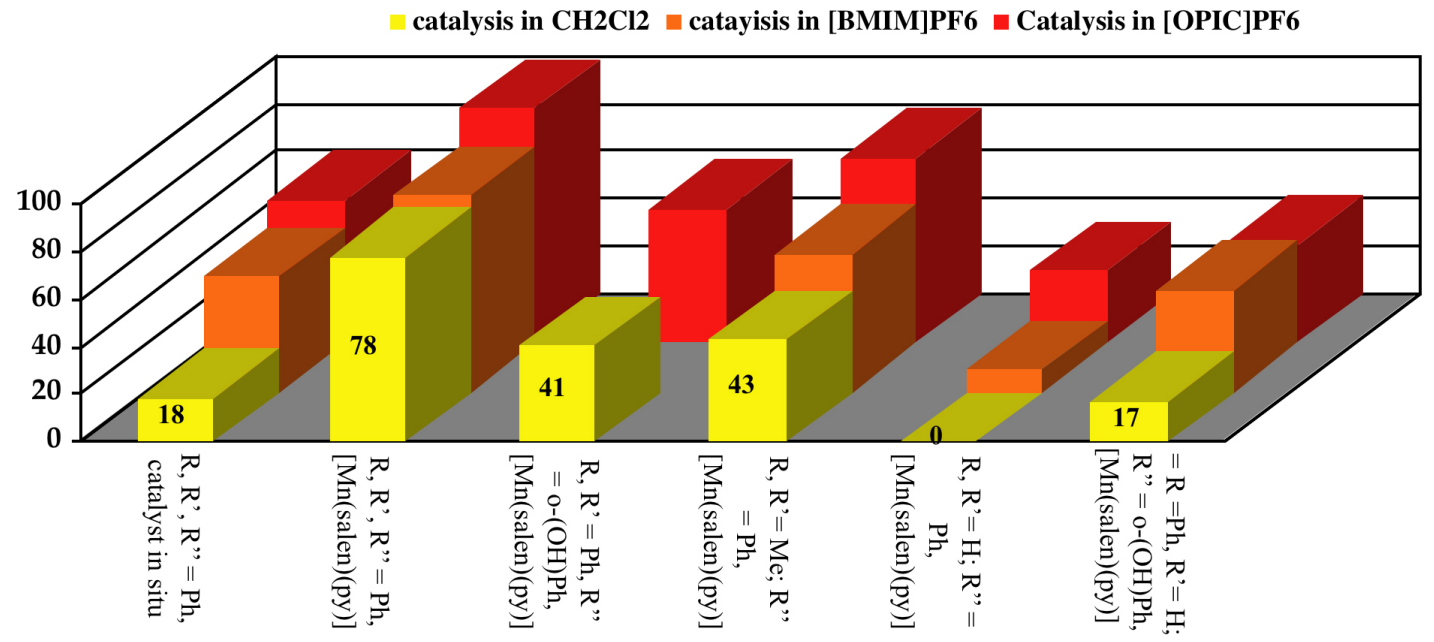

Fig. (1). Oxidative cleavage of benzopinacol (entries 1-3), pinacol (entry 4), ethylenglycol (entry 5) and hydrobenzoin (entry 6) in $\mathrm{CH}_{2} \mathrm{Cl}_{2}$, $\left[\mathrm{BMIM}_{\mathrm{PF}_{6}}\right.$ and in $[\mathrm{OPIC}] \mathrm{PF}_{6}$, catalyzed by $5 \mathrm{~mol} \%[\mathrm{Mn}(\mathrm{salen})(\mathrm{Py})] \mathrm{OAc}$. Yields are for isolated material after FC and the results are an average of 2 runs.

yield

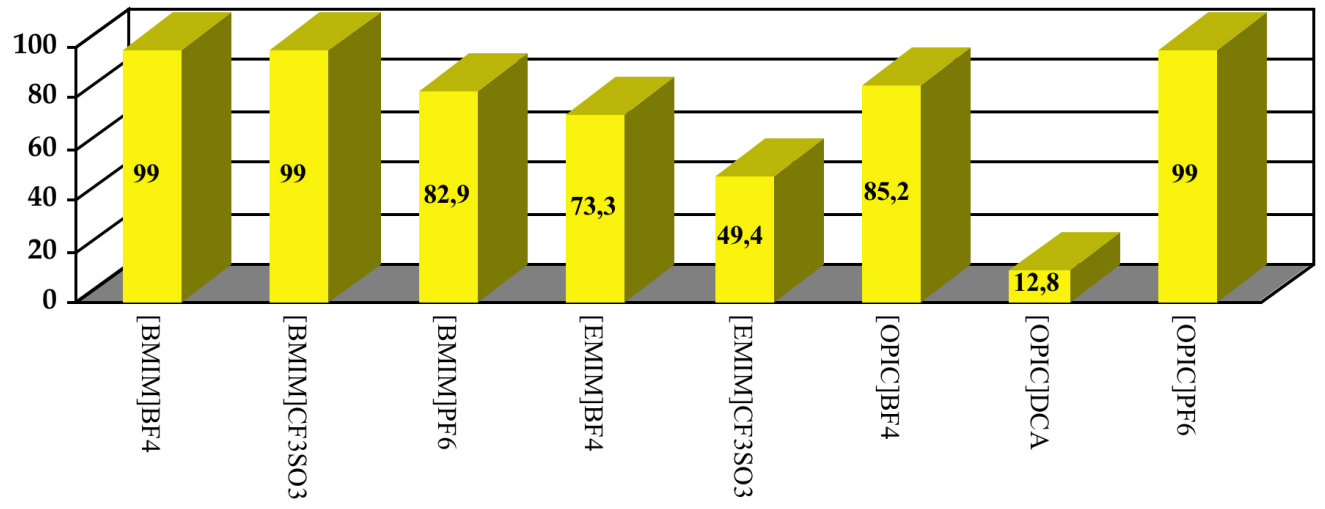

Fig. (2). Oxidative Cleavage of benzopinacol catalyzed by $[\mathrm{Mn}(\mathrm{salen})(\mathrm{Py})] \mathrm{OAc}$ dissolved in a range of ionic liquids, with air as oxidant. Yields are for isolated material after FC and the results are an average of 2 runs.

oxygen) as oxidant is almost effective in ionic liquids, with yields generally more than $80 \%$. Only in the case of the reaction carried out in [EMIM]-based ionic liquids (entries 4 and 5, Fig. 2) the yield is lower $(73,3 \%$ in [EMIM]BF 4 and $49,4 \%$ in [EMIM] $\mathrm{CF}_{3} \mathrm{SO}_{3}$ ), showing that also the cationic precursor of the ionic liquid can play an important role in this catalytic reaction. In the case of the reaction in [OPIC]DCA (entry 7, Fig. 2), the solubility of the catalyst $[\mathrm{Mn}($ salen $)(\mathrm{Py})](\mathrm{OAc})$ is not really good in this ionic liquid, and therefore the reaction is not efficient (yield only 12,8\%).

In order to widen the use of these ionic liquids, the catalyst/ionic liquid system has been recycled (Fig. 3). It is interesting to note, that the recycled system is even more active that the previous used catalyst. Using the ionic liquids based on [BMIM] salts (entries 1,2) the yields are almost quantitative. In the case of [EMIM] (Entries 3,4) and [OPIC]-based ionic liquids (entries 5,6) the yields increase in the recycled experiment. This perhaps indicates that the formation of the active species of the catalyst (induction time) plays possibly an important function in the optimization of the catalytic reaction. Also in the worst example with [OPIC]DCA, the yield increases from 12,8\% to $21,9 \%$ (always isolated yields). The possibility to extend the recycle experiments of the system catalyst/ionic-liquid with additional runs is limited by the presence of the corresponding acid, formed from the co-reductant benzaldehyde: the use of hexane/ethylacetate $(9: 1)$ to extract the products is not really efficient for the formed benzoic acid and residue of the acid remained in the ionic liquid (control with TLC and GC-MS). Therefore, the excess of the acid involve a more viscous reaction mixture, which after the first recycled experiment didn't allow further recycling processes.

Although different groups have reported the oxidation cleavage of diols catalyzed by transition metal complexes, a really satisfactory mechanism that explains the interaction 
Yields of 1st run $\square$ Yields of recycled IL/cat

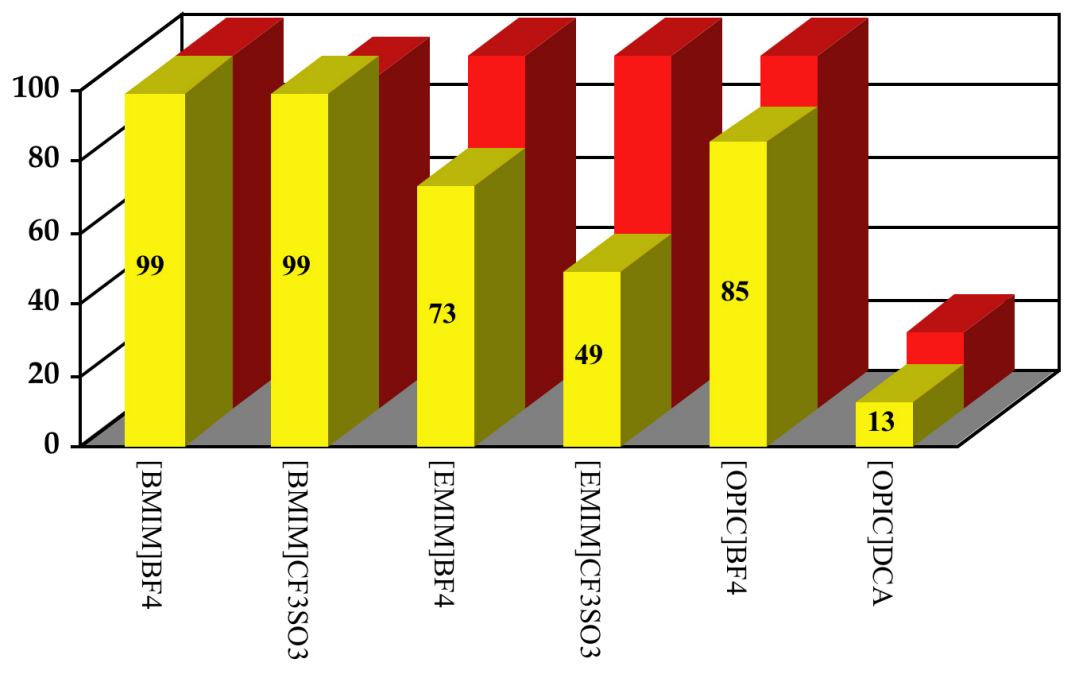

Fig. (3). Oxidative Cleavage of benzopinacol catalyzed by [Mn(salen)(Py)]OAc in IL or by recycled IL/catalyst-system, with air as oxidant. Yields are for isolated material after FC and the results are an average of 2 runs.

between the substrates and the catalyst has still to be detailed. Several groups $[15,25,26]$ proposed that the reaction is catalyzed by an oxomanganese active specie, which is formed by a sacrificial aldehyde that must be present in stoichiometrical quantities (or in excess). Using DFT (Density Functional Theory) and UHF (Unrestricted Hartee-Fock) calculations, we tried to understand how the oxo manganese complex catalyses the oxidation cleavage of the diols. To model this reaction, the use of a B3LYP/LANL2DZ level of theory has been established [27-30]. In order to simplify the computational model, the preliminary DFT studies [23] were made with ethylenglycol as the substrate, the oxomanganese $(\mathrm{V})$ chloride as the catalyst (the neutral species to simplify the underlying calculations) and a simplified salen ligand (without the lateral aromatic ring of the salicylaldehyde) [29-32]. The first step to get the reaction approximation's coordinates of the substrate was the mapping of the electrostatic potential of the diol and the manganese complex (Fig. 4). The substrate and the complex showed a negative potential of almost equal magnitude around their active oxygen atoms $(\mathrm{ESP}=-0.03$ ). Thus, the oxygen-oxygen approach to form a peroxide intermediate as in an iron porphyrine compound is almost impossible owing to electrostatic repulsion.

The diol approaching the oxomanganese complex formed an intermediate with multiplicity 5 , in which a proton from the diol was transferred to the manganese complex (Fig. 5, left; $\mathrm{C}$-C distance in the diol $=1,586 \AA$ ). A local minimum was found when both protons of the diol had been donated. This geometry involved the formation of two molecules of formaldehyde and a molecule of an aqua-manganese complex (Fig. 5, right). Finally, all transition states and minima were verified by hessian calculations, showing the correct amount of imaginary frequencies.

In order to understand the manganese-catalyzed oxidation of diols by evaluating the catalytic cycle, both IRC (Intrinsic reaction coordinate) and PCM (Polarized
Continuum Model) calculations (to include solvent effects) have been considered. We also wanted to evaluate the effect of the axial ligand, changing from chloride to pyridine, since the later showed to increase reaction rates and activities of the catalyst [23]. To achieve this goals we decided to change the computational methodology towards the LANL2MB/ B3LYP level of theory as it is implemented in Gaussian 03 [33]. The smaller size of this basis set allows exploring the reaction path more extensively, while maintains an adequate description of the chemical system. Methodologically, we started finding the different transition states and confirming them by frequency calculations. A subsequent IRC study allowed the location of both reactant and product complexes, associated with the calculated transition states. Additionally, both reactant and products obtained by IRC were optimized to ensure that they were true local minima.

If the axial ligand is changed by a pyridine ligand, the activation energy is lower (Scheme $\mathbf{3}$ ): the effect of the axial ligand is crucial in these type of catalysis, and explains the experimental results, where the pyridine is necessary also in the in situ-catalysis [23]. The reaction path was confirmed through IRC calculations from which both the reactant and product complex energies were also established. This reaction step was calculated for both pyridine and chloride containing complexes obtaining activation energies of 66.5 and $108.5 \mathrm{~kJ} / \mathrm{mol}$ respectively. The high energy barrier decrease with the inclusion of pyridine as axial ligand and can be explained by the stabilizing effect generated by the $\pi$-accepting capability of this axial ligand [34]. As it is expected, the reaction is in general exothermic, with an energy change of $-127.37 \mathrm{KJ} / \mathrm{mol}$ for the pyridine complex and $-80.38 \mathrm{~kJ} / \mathrm{mol}$ for the chloride complex. This means that the reaction is both favored kinetically and thermodynamically when chloride is substituted by pyridine, which is very important because this step (Scheme $\mathbf{3}, \mathbf{3} \Rightarrow$ TS2 $\Rightarrow$ 4) is rate determining for the catalytic cycle. To further improve our understanding of the catalytic cycle, we also studied the generation of the oxomanganese specie (2). Even though it 

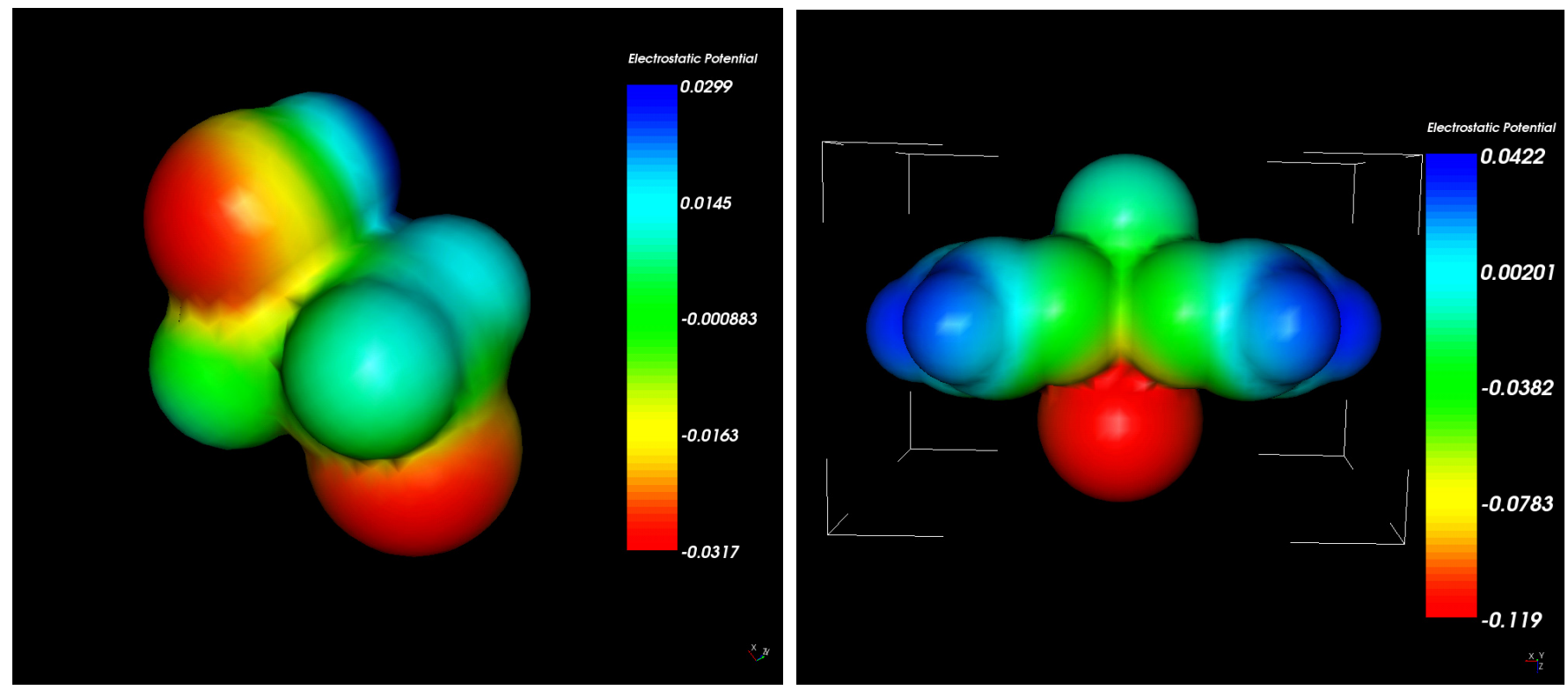

Fig. (4). Mapping of the electrostatic potential of the diol (left) and the simplified chloro-manganese(III) complex (right).

has been proposed that the complex is formed by a radical mechanism $[15,26]$, it is possible that the complex is produced via a non-radical reaction of a molecular oxygen manganese(salen) complex with the sacrificial aldehyde. This would also explain why the reaction is not hindered in ionic liquids, which would be prompt to happen if it were highly dependent on radical formation. A transition state was found (Scheme 4, TS1), linked (via IRC) with the reaction path from the molecular oxygen complex to the oxomanganese complex formation. The activation energy for this step was determined to be 42.94 for the pyridine- and $26.38 \mathrm{KJ} / \mathrm{mol}$ for the Cl-complex. The lower activation energy for the chloride complex can be explained by the $\pi$-donor character of the anionic chloride ligand, making the transition towards $\mathrm{Mn}(\mathrm{V})$ easier. This is also observed by the overall energetics of this step, which are -59.3 for the pyridine and $-83.9 \mathrm{KJ}$ for the Cl-containing complex. Consequently, this step is kinetically and thermodynamically easier with the Cl-complex. Finally, the calculated catalytic cycle is showed in Scheme 4: in the cycle, the co-reductant in the presence of oxygen allows the formation of the oxo specie $(\mathbf{1} \Rightarrow \mathbf{T S 1} \Rightarrow \mathbf{2})$, which can interact with the substrate, to form the intermediate $(\mathbf{3})$. The concerted interaction between the diol and the catalyst (TS2) consents the formation of the aqua-complex (4) and two molecules of aldehydes. After the elimination of the water, the catalyst is reformed and the cycle is closed.

Moreover, both intermediates and transition state (Fig. 5, $\mathrm{C}-\mathrm{C}$ distance in transition state $($ TS2) $=1,736 \AA$ ) showed high areas of charge separation in the electrostatic potential maps. This suggests that the use of solvents with high dielectric constants as well as the use of cationic manganese complexes would cause further stability of the intermediates and the transition states. Therefore, we tried to include solvent and ionic liquid effects using the PCM model. However, this approach failed to give realistic results due to the simplicity of the computational model. Since in PCM

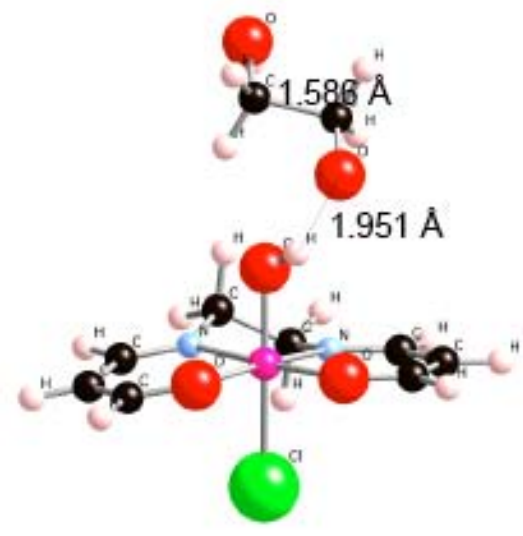

(3)

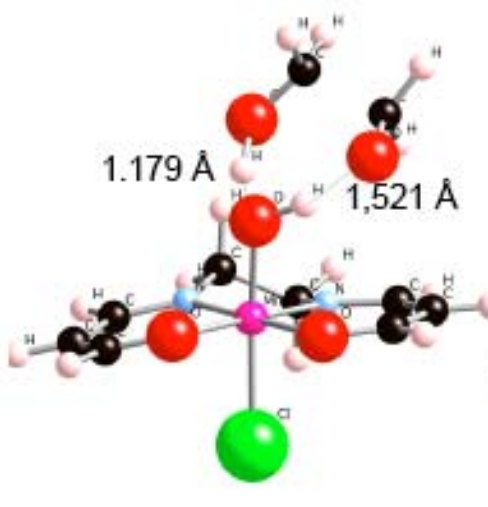

(TS2)

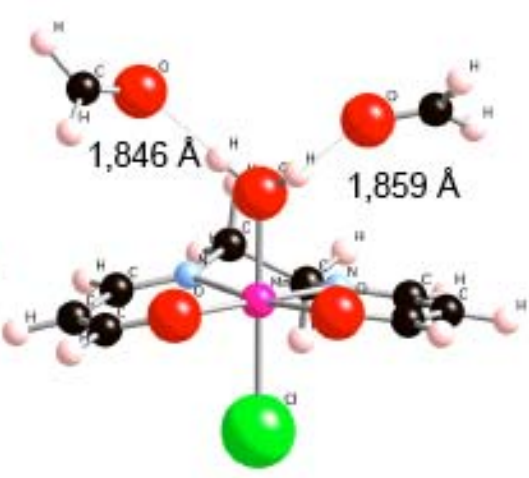

(4)

Fig. (5). Calculated interaction catalyst-substrates: intermediates (3) and (4) and transition state TS2. Illustration generated using CrystalMaker ${ }^{\circledR}$. 


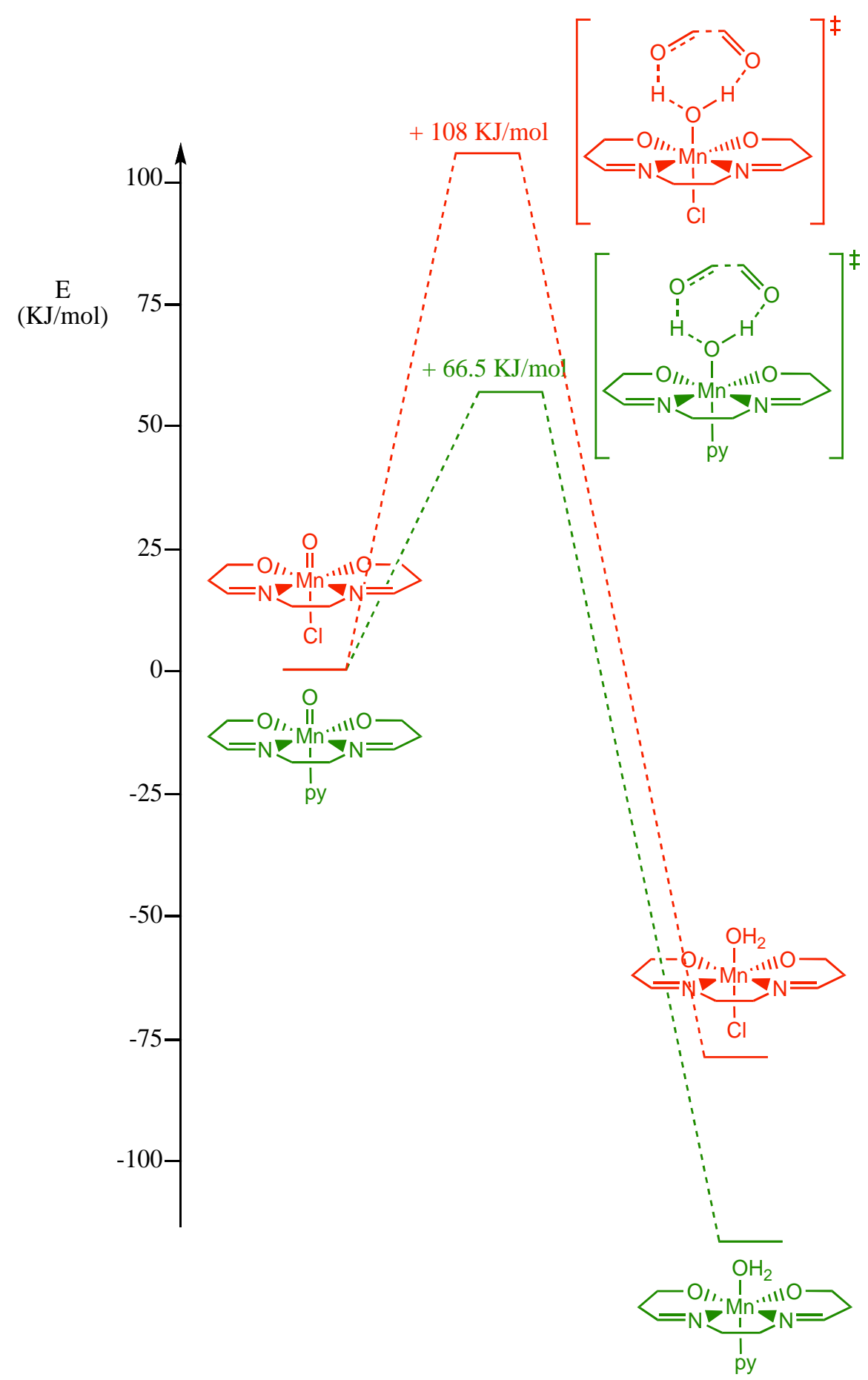

Scheme 3. Calculated activation energy for $(3 \Rightarrow$ TS2 $\Rightarrow 4)$, the rate determining step: effect of the axial ligand (multiplicity $=1)$.

there is no distinction between different "solvent entities" and the solvent is treated as an homogeneous continuum of constant polarity, the different molecules present in an ionic liquid with their different charges is not at all represented. Since the transition states have significant dipole moments, it is expected that the solvent would arrange in a particular way around the molecule, which can be simulated only by the explicit inclusion of solvent molecules. The inclusion of a solvent molecule (by PCM model) showed the stabilization of reactants and products much more than the transition state structures, which gave an overall increase of activation energies: this is the opposite of what is experimentally observed.

Since the PCM approach did not give satisfactory results, we decided to calculate an ionic liquid explicitly by using the approach described by Bessac et al. [35], which gives accurate results and is computationally efficient. We used an Oniom level calculation DFT/MM in which 12 ionic pairs of the $N^{\prime}, N$-dimethylimidazolium tetrafluoroborate were treated as ionic liquid, with a low level of theory described by the UFF force field, while the catalyst-reagent system was treated with a higher level of theory described by the 


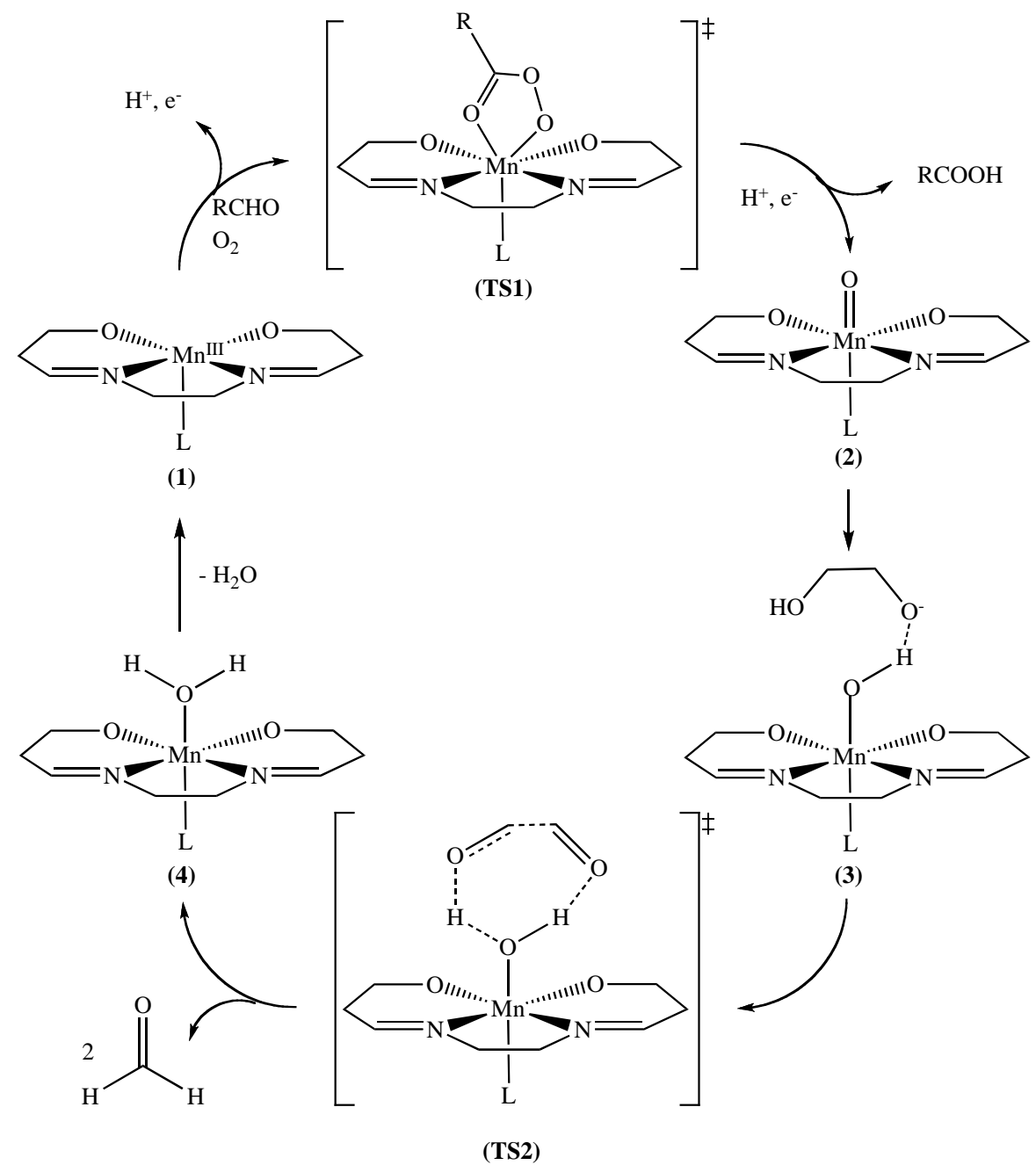

Scheme 4. Calculated catalytic cycle of the oxidative cleavage of vic-diols catalyzed by $[\mathrm{Mn}(\mathrm{salen})(\mathrm{L})]^{+}$complexes.

LANL2MB basis set using the B3LYP density functional. Ionic pairs were placed symmetrically around the catalyst for the starting geometries. Ionic pair molecule geometries were previously optimized at a B3LYP/LANL2DZ level of theory. The calculation required the explicit computation of second derivatives on every optimization step, since the potential energy surface appeared to be significantly flat. The optimization to a transition state, starting from the transition state geometry previously determined in the gas phase, led to the system shown in Fig. (6). The UFF force field failed to describe the methyl carbon - nitrogen bond accurately (as the methyl group is not coplanar with the imidazole ring in most ionic pairs), although overall electrostatic contributions are in accordance with what would be expected from this type of ionic systems. There were also significant changes in the transition state's geometrical configuration, which exhibits an important torsion of the pyridine ligand, which ends up being totally aligned with the $\mathrm{O}$ and $\mathrm{N}$ atoms of the salen moiety (it was completely parallel to these atoms in the gas phase). This torsion of pyridine causes an overall rotation of the glycol's location relative to the salen-ring. An IRC calculation was performed from this transition state in order to complete the reaction step in the presence of the explicit ionic liquid. Minimization from the two extremes of the IRC calculation afforded both reagent and product geometries
(Fig. 7, Intermediate (3)). The energy difference between the reagents and the transition state was determined to be 83.11 $\mathrm{kJ} / \mathrm{mol}$, which is greater than the previously calculated energy of $66.5 \mathrm{~kJ} / \mathrm{mol}$. However, the pyridine conformational change is especially responsible for this. Removing solvent molecules and optimizing again to a transition state in the gas phase leads to an energy increase of almost $60 \mathrm{KJ} / \mathrm{mol}$, which quickly shifts to the previous conformation of the pyridine ligand, meaning that there is a significant stabilization of that conformation in the ionic liquid. The previously achieved low energy of $66.5 \mathrm{~kJ} / \mathrm{mol}$ seems to be artificial and valid only in the gas phase. This conformation is unstable in the presence of solvent molecules: consequently, the lowest possible energies are associated with the newly found conformation, which is stable in the presence of solvent molecules. This stabilization in ionic liquids is absent for the chloride complex: in this case, the activation energy increases slightly to $118 \mathrm{KJ} / \mathrm{mol}$, with no overall important geometrical changes. Due to ionic liquid stabilization, also the overall energy differences between reagents and products became more favorable (around $10 \mathrm{KJ} / \mathrm{mol}$ ).

The study of this reaction step in the presence of an explicit ionic liquid showed the stabilizing effect of the solvent on a very unstable conformation of the pyridine ring 
in the gas phase and hence, a much lower activation energy than that which would have been expected from the same conformation without solvent molecules. It also showed that this effect is clearly seen for the cationic complex (with pyridine as axial ligand), whilst the chloride complex (neutral) didn't show important stabilizing effects in the presence of the polar solvent. In conclusion, ionic liquids may help stabilizing cationic or anionic transition states significantly, while they have a much lower influence on neutral catalysts.

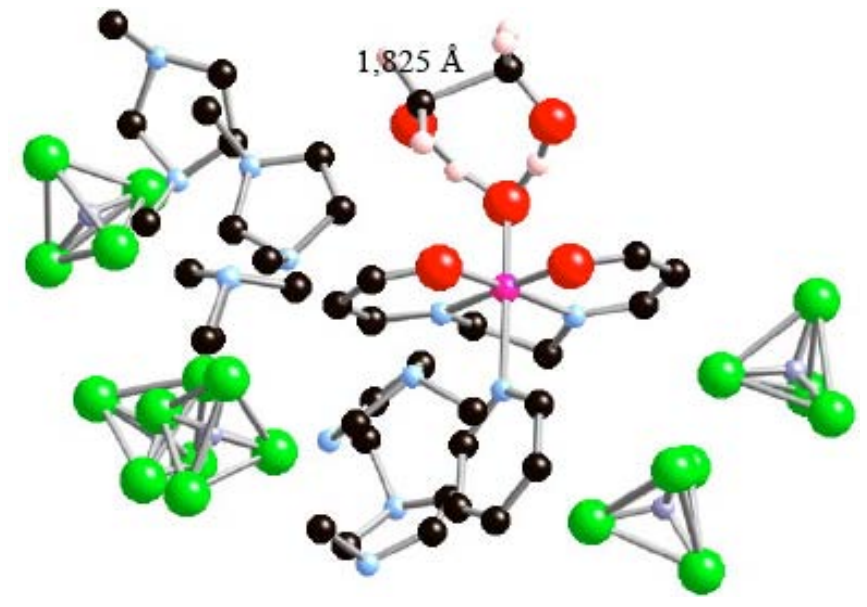

Fig. (6). Calculated interaction catalyst-substrates in IL for the transition state TS2. (Oniom level calculation DFT/MM with 12 ionic pairs of the $\mathrm{N}^{\prime}, \mathrm{N}$-dimethylimidazolium tetrafluoroborate as ionic liquid: only 5 pairs are presented for clarity. Most hydrogen atoms are also omitted for clarity. Illustration generated using CrystalMaker ${ }^{\circledR}$.

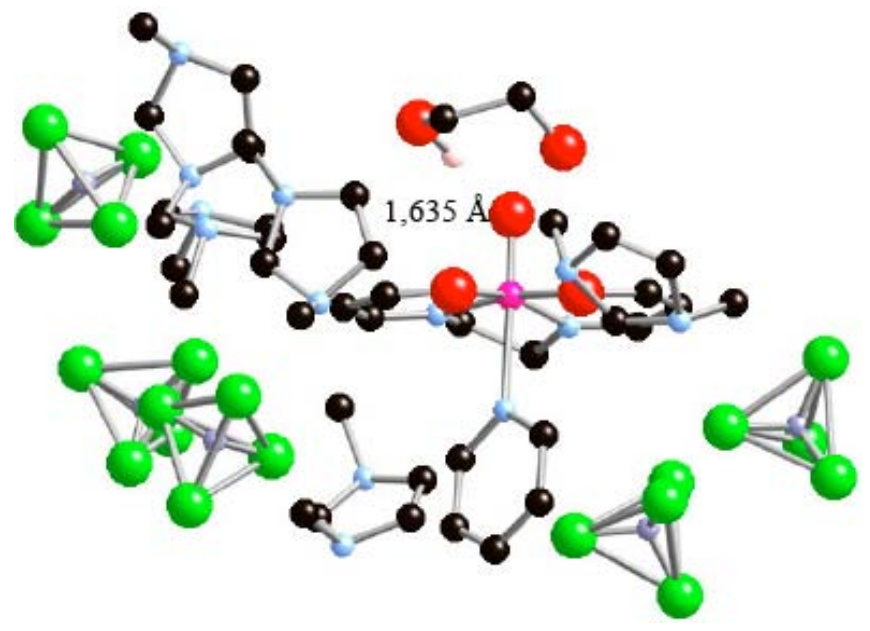

Fig. (7). Calculated interaction catalyst-substrates in IL for the intermediate (3). (Oniom level calculation DFT/MM with 12 ionic pairs of the $\mathrm{N}^{\prime}, \mathrm{N}$-dimethylimidazolium tetrafluoroborate as ionic liquid: only 5 pairs are presented for clarity. Most hydrogen atoms are also omitted for clarity. Illustration generated using CrystalMaker $^{\circledR}$

\section{CONCLUSIONS}

The complex $[\mathrm{Mn}($ salen$)(\mathrm{Py})] \mathrm{OAc}$ presents catalytic activity for the oxidative $\mathrm{C}$ - $\mathrm{C}$ bond cleavage of vic-diols with oxygen as oxidant and an aldehyde as co-reductant. Ketones from steric hindered substrates like 1,1,2,2-tetraphenyl-1,2-ethanediol (benzopinacol) have been formed (with yields up to $99 \%, 2 \mathrm{~h}$ at $60{ }^{\circ} \mathrm{C}$ )), showing better results than the previously reported systems based on manganese(III) catalysts.

The use of ionic liquids as solvents in this reaction allows us to improve the results considerably, increasing the yield up to $60 \%$. These results corroborate the positive effect of the use of ionic liquids with cationic catalysts. DFT calculations let us clarify the possible interaction between the substrates and the catalyst. After this study and for the first time, there is a complete cycle that explains the Mn-catalyzed oxidative cleavage of diols. The mechanism explains the formation of the oxomanganese complex, the role of oxygen, the diol oxidation transition state and the regeneration of the catalyst by water dissociation. The proposed mechanism also gives us a good understanding about why this reaction works extremely well for manganese(salen)-complexes, with pyridine ligands, in ionic liquids. Further kinetic studies to verify the catalytic cycle are in progress.

\section{ACKNOWLEDGEMENTS}

The authors thank Prof. A. Togni (ETH Zürich, Switzerland) for the donation of the ionic liquids, the use of the parallel organic synthesizer Quest 210 and the computer's time for the theoretical study. Prof. A. Reyes and Prof. E. Daza (Universidad Nacional de Colombia) are also acknowledged for the assistance in the calculations.

\section{REFERENCES}

[1] Shing, T.K.M. Glycol Cleavage Reactions. In: Trost, B. M.; Fleming, I., Eds. Comprehensive Organic Synthesis, Pergamon Press: Oxford, 1991, Vol. 7, pp. 703-716.

[2] Caron, S.; Dugger, R. W.; Gut Ruggeri, S.; Ragan, J. A.; Brown Ripin, D. H. Large-scale oxidations in the pharmaceutical industry. Chem. Rev., 2006, 106 (7), 2943-89.

[3] Meunier, B. Biomimetic Oxidations Catalyzed by Transition Metal Complexes. Imperial College Press: London, 2000.

[4] Schmid, C. R.; Bryant, J. D.; Dowlatzedah, M.; Phillips, J. L.; Prather, D. E.; Schantz, R. D.; Sear, N. L.; Vianco, C. S. Synthesis of 2,3-O-isopropylidene-D-glyceraldehyde in high chemical and optical purity: observations on the development of a practical bulk process. J. Org. Chem., 1991, 56 (12), 4056-58.

[5] Nair, V.; Deepthi, A. Cerium(IV) ammonium nitrate a versatile single-electron oxidant. Chem. Rev., 2007, 107(5), 1862-91.

[6] Felthouse, T.R. Oxidation with encapsulated co-catalyst. US Patent 4959494, 1986.

[7] Barton, D. H. R.; Motherwell, W. B.; Stobie, A. A catalytic method for a-glycol cleavage. J. Chem. Soc. Chem. Commun., 1981, 1232-33.

[8] Greenspan, F. P.; Woodburn, H. M. The Action of Silver-catalyzed Persulfate on 1,2-Glycols. J. Am. Chem. Soc., 1954, 76(24), 6345-49.

[9] Okamoto, T.; Sasaki, K.; Oka, S. Biomimetic oxidation with molecular oxygen. Selective carbon-carbon bond cleavage of 1,2-diols by molecular oxygen and dihydropyridine in the presence of iron-porphyrin catalysts. J. Am. Chem. Soc., 1988, 110(4), 1187-96.

[10] Wasserscheid, P.; Keim, W. Ionic liquids - New "solutions" for transition metal catalysis. Angew. Chem. Int. Ed., 2000, 39(21), 3773-89.

[11] Holbrey, J. D.; Seddon, K. R. Ionic liquids. Clean Prod. Proc., 1999, l(4), 223-36.

[12] Baleizao, C.; Garcia, H. Chiral salen complexes: an overview to recoverable and reusable homogeneous and heterogeneous catalysts. Chem. Rev., 2006, 106 (9), 3987-4043.

[13] Zhang, W.; Jacobsen, E. N. Asymmetric olefin epoxidation with sodium hypochlorite catalyzed by easily prepared chiral manganese(III) salen complexes. J. Org. Chem., 1991, 56(7), 2296-98. 
[14] Zhang, W.; Loebach, J. L.; Wilson, S. R.; Jacobsen, E. N. Enantioselective epoxidation of unfunctionalized olefins catalyzed by salen manganese complexes. J. Am. Chem. Soc., 1990, 112(7), 2801-03.

[15] Mukaiyama, T.; Yamada, T. Recent advances in aerobic oxygenation. Bull. Chem. Soc. Jpn., 1995, 68(1), 17-35.

[16] Conrad Zhang, Z. Catalysis in Ionic Liquids. Adv. Catal., 2006, 49, 153-237.

[17] Welton, T. Ionic liquids in catalysis. Coord. Chem. Rev., 2004, 248 (21-24), 2459-77.

[18] Dupont, J.; de Souza, R. F.; Suarez, P. A. Z. Ionic liquid (molten salt) phase organometallic catalysis. Chem. Rev., 2002, 102(10), 3667-91.

[19] Parvulescu, V. I.; Hardacre, C. Catalysis in ionic liquids. Chem. Rev., 2007, 107(6), 2615-65.

[20] Emmanuvel, L.; Shaikh, T.; Sudalai, A. NaIO4/LiBr-mediated Diastereoselective Dihydroxylation of Olefins:A Catalytic Approach to the Prevost-Woodward Reaction. Org. Lett., 2005, 7, 5071-74.

[21] Bachmann, W. Benzopinacole. Org. Synth., Coll., 1943, Vol. 2, 71.

[22] Adams, R.; Adams, E.V. Pinacol hydrate. Org. Synth. Coll., 1941, Vol. $1,459$.

[23] Riaño, S.; Fernández, D.; Fadini, L. Oxidative cleavage of vic-diols catalyzed by manganese(III) complexes in ionic liquids. Catal. Commun., 2008, 9, 1282-85.

[24] Mukaiyama, T. New possibilities in organic synthesis. Aldrichim. Acta, 1996, 29 (3), 59-76.

[25] Barroso, S.; Blay, G.; Fernandez, I.; Pedro, J. R.; Ruiz-Garcia, R.; Pardo, E.; Lloret, F.; Munoz, M. C. Chemistry and reactivity of mononuclear manganese oxamate complexes: Oxidative carbon-carbon bond cleavage of vic-diols. J. Mol. Catal. A Chem., 2006, 243 (2), 214-20.

[26] Sibbons, K.F.; Shastri, K.; Watkinson, M. The application of manganese complexes of ligands derived from 1,4,7-triazacyclononane in oxidative catalysis. Dalton Trans., 2006, 645-61.

[27] Hay, P. J.; Wadt, W. R. Ab initio effective core potentials for molecular calculations. Potentials for the transition metal atoms Sc to Hg. J. Chem. Phys., 1985, 82(1), 270-83.

[28] Lee, C.; Yang, W.; Parr, R. G. Development of the Colle-Salvetti correlation-energy formula into a functional of the electron density. Phys. Rev. B, 1988, 37(2), 785.
[29] Cavallo, L.; Jacobsen, H. Toward a catalytic cycle for the Mn-salen mediated alkene epoxidation: A computational approach. Inorg. Chem., 2004, 43(6), 2175-82.

[30] Cavallo, L.; Jacobsen, H. Transition metal mediated epoxidation as test case for the performance of different density functionals: A Computational Study. J. Phys. Chem. A, 2003, 107(28), 5466-71.

[31] Cavallo, L.; Jacobsen, H. Electronic effects in (salen)Mn-based epoxidation catalysts. J. Org. Chem., 2003, 68(16), 6202-07.

[32] Cavallo, L.; Jacobsen, H. Manganese-salen complexes as oxygen-transfer agents in catalytic epoxidations - A density functional study of mechanistic aspects. Eur. J. Inorg. Chem., 2003, $5,892-902$.

[33] Gaussian03, Revision C.03., Frisch, M. J.; Trucks, G. W.; Schlegel, H. B.; Scuseria, G. E.; Robb, M. A.; Cheeseman, J. R.; Montgomery, Jr., J. A.; Vreven, T.; Kudin, K. N.; Burant, J. C.; Millam, J. M.; Iyengar, S. S.; Tomasi, J.; Barone, V.; Mennucci, B.; Cossi, M.; Scalmani, G.; Rega, N.; Petersson, G. A.; Nakatsuji, H.; Hada, M.; Ehara, M.; Toyota, K.; Fukuda, R.; Hasegawa, J.; Ishida, M.; Nakajima, T.; Honda, Y.; Kitao, O.; Nakai, H.; Klene, M.; Li, X.; Knox, J. E.; Hratchian, H. P.; Cross, J. B.; Bakken, V.; Adamo, C.; Jaramillo, J.; Gomperts, R.; Stratmann, R. E.; Yazyev, O.; Austin, A. J.; Cammi, R.; Pomelli, C.; Ochterski, J. W.; Ayala, P. Y.; Morokuma, K.; Voth, G. A.; Salvador, P.; Dannenberg, J. J.; Zakrzewski, V. G.; Dapprich, S.; Daniels, A. D.; Strain, M. C.; Farkas, O.; Malick, D. K.; Rabuck, A. D.; Raghavachari, K.; Foresman, J. B.; Ortiz, J. V.; Cui, Q.; Baboul, A. G.; Clifford, S.; Cioslowski, J.; Stefanov, B. B.; Liu, G.; Liashenko, A.; Piskorz, P.; Komaromi, I.; Martin, R. L.; Fox, D. J.; Keith, T.; Al-Laham, M. A.; Peng, C. Y.; Nanayakkara, A.; Challacombe, M.; Gill, P. M. W.; Johnson, B.; Chen, W.; Wong, M. W.; Gonzalez, C.; Pople, J. A.; Gaussian, Inc., Wallingford CT, 2004.

[34] Srinivasan, K.; Michaud, P.; Kochi, J. K. Epoxidastion of olefins with cationic (salen)Mn(III) complexes. The modulation of catalytic activity by substituents. J. Am. Chem. Soc., 1986, 108(9), 2309-20.

[35] Bessac, F.; Maseras, F. DFT modeling of reactivity in an ionic liquid: How many ion pairs? J. Comput. Chem., 2007, 29(6), 892-99.

(C) Fernández et al.; Licensee Bentham Open.

This is an open access article licensed under the terms of the Creative Commons Attribution Non-Commercial License (http://creativecommons.org/licenses/by-nc/ $3.0 /$ ) which permits unrestricted, non-commercial use, distribution and reproduction in any medium, provided the work is properly cited. 